\title{
A Novel Femtosecond Laser System for Attosecond Pulse Generation
}

\author{
Jianqiang Zhu, Xinglong Xie, Meizhi Sun, Qunyu Bi, and Jun Kang \\ National Laboratory on High Power Laser and Physics, Shanghai Institute of Optics and Fine Mechanics, Chinese Academy of Sciences, \\ Number 390, Qinghe Road, Jiading, Shanghai 201800, China \\ Correspondence should be addressed to Xinglong Xie, xiexl329@mail.shcnc.ac.cn
}

Received 15 March 2011; Revised 22 September 2011; Accepted 2 October 2011

Academic Editor: Ci-Ling Pan

Copyright () 2012 Jianqiang Zhu et al. This is an open access article distributed under the Creative Commons Attribution License, which permits unrestricted use, distribution, and reproduction in any medium, provided the original work is properly cited.

\begin{abstract}
We report a novel ultrabroadband high-energy femtosecond laser to be built in our laboratory. A 7-femtosecond pulse is firstly stretched by an eight-pass offner stretcher with a chirp rate $15 \mathrm{ps} / \mathrm{nm}$, and then energy-amplified by a two-stage optical parametric chirped pulse amplification (OPCPA). The first stage as preamplification with three pieces of BBO crystals provides the majority of the energy gain. At the second stage, a YCOB crystal with the aperture of $\sim 50 \mathrm{~mm}$ is used instead of the KDP crystal as the gain medium to ensure the shortest pulse. After the completion, the laser will deliver about $8 \mathrm{~J}$ with pulse duration of about 10 femtoseconds, which should be beneficial to the attosecond pulse generation and other ultrafast experiments.
\end{abstract}

The attosecond pulse has been of interest in recent years and proved to be a powerful tool in studying the ultrafast phenomena, such as the chemical/biological transformations occurring on the femtosecond timescale and the evolution of the dynamics of the electrons in atoms or molecules irradiated by intense lasers. There are two ways to generate an attosecond pulse/pulse train. One is through the nonlinear processes of the superposition of high order harmonics generated in the laser-gas atom interactions in which the laser intensity should be low so as to avoid the ionization of atoms. Up to now, the laser intensity is no more than $10^{16} \mathrm{~W} \mathrm{~cm}^{-2}$ and the efficiency of harmonic emission from the atoms is low. The other way is to generate the high order harmonics from the dense surface of a plasma created by the high-intensity femtosecond laser, which is promising for the intense attosecond pulse generation. Recent experiments and simulations have shown that attosecond pulses with high conversion efficiency, high photon energy, and excellent divergence can be generated [1-4].

The efficiency of harmonic generation from the laserplasma surface relies on the laser intensity. In the experiments for the brilliant harmonic generation from the surface, the laser has energy of $0.1 \mathrm{~J}$ to less than $10 \mathrm{~J}$ with a pulse duration of $30-50 \mathrm{fs}$ [5]. In this paper, we report a novel ultrabroadband ultrashort high-energy laser system based on optical parametric chirped pulse amplification (OPCPA). After completion, the laser can deliver $8.0 \mathrm{~J}$ with pulse width of about $10 \mathrm{fs}$, which is much shorter than those used in the previous intense attosecond pulse generation experiments. The laser should be beneficial to the attosecond pulse generation and other ultrafast experiments.

OPCPA has been applied and becomes promising for constructing high-intensity lasers [5-9], particularly with the invention of new nonlinear crystals such as YCOB $\left(\mathrm{YCa}{ }_{4} \mathrm{O}\left(\mathrm{BO}_{3}\right)_{3}, \mathrm{YCOB}\right)$ [10-12]. Compared with other nonlinear crystals commonly utilized in OPCPA systems, YCOB crystal has several prominent properties: high damage threshold, moderate thermal conductivity, and nonlinear coupling [13]. It has been shown that the size of YCOB crystal can be as large as $7.5 \mathrm{~cm}$ in diameter and $25 \mathrm{~cm}$ in length [14], much larger than those currently used, such as $\mathrm{LBO}$ and $\mathrm{BBO}$ crystals. Chekhlov et al. reported their OPCPA laser system which used large aperture KDP crystals for power amplification. Their laser delivers output energy up to $35 \mathrm{~J}$ and pulse width of $84 \mathrm{fs,} \mathrm{[15].} \mathrm{To} \mathrm{our} \mathrm{knowledge,}$ YCOB crystal has not been used in OPCPA laser systems with wavelength centered at $808 \mathrm{~nm}$ and output energy up to several joules and pulse width of less than $10 \mathrm{fs}$. 


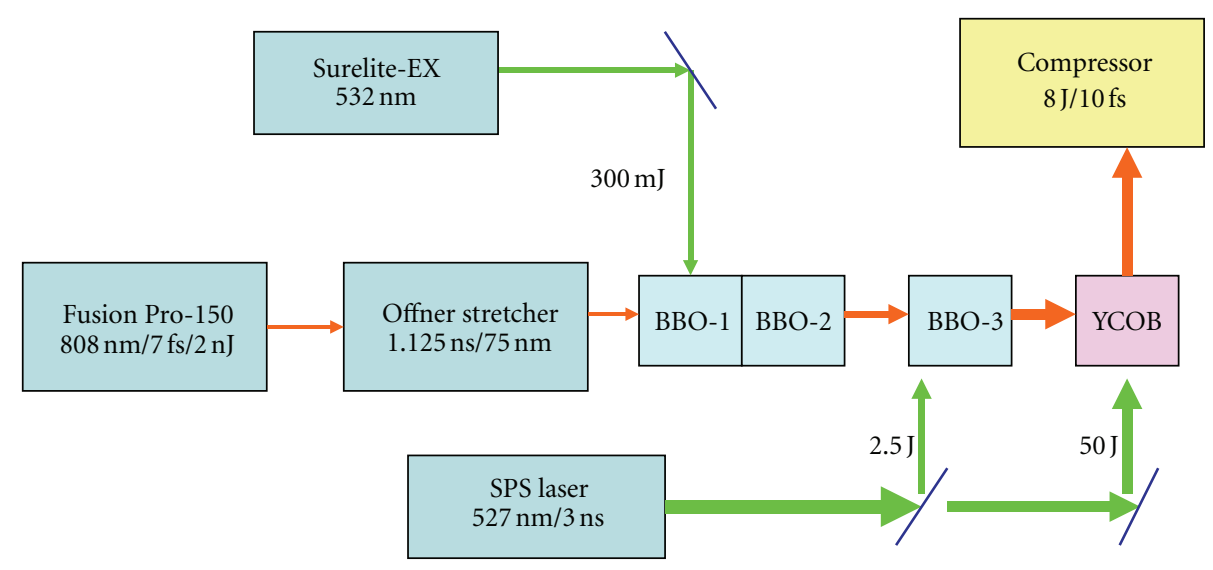

Figure 1: Optical schematic layout of OPCPA.

To design the OPCPA-based ultrashort laser system which can deliver several joules within about $10 \mathrm{fs}$, we use YCOB instead of KDP or DKDP crystal as the gain medium for power amplification stage. Because YCOB has nonlinear coefficient four times of that of KDP and DKDP, it can provide broad spectral gain bandwidth. As indicated in Figure 1, our laser system consists of five parts: a signal laser pulse, an Offner stretcher, a multistage optical parametric amplifier (OPA), a compressor, and a pump laser system. The oscillator provides signal pulses of $7 \mathrm{fs}$ in time duration and $2 \mathrm{~nJ}$ in energy. The signal pulses are chirped to $15 \mathrm{ps} / \mathrm{nm}$ by an eight-pass Offner stretcher with 10 percent transmission efficiency, and the energy of chirped signal pulse injected into OPA chain is measured to be about $0.2 \mathrm{~nJ}$. The OPCPA amplification chain is composed of two stages. The first stage, as a preamplification, includes three pieces of $\mathrm{BBO}$ crystals that provide the majority of the energy gain. The pump pulses at $532 \mathrm{~nm}$ with $300 \mathrm{~mJ}$ for the first two pieces of BBO crystals are from a commercial single longitudinal mode $\mathrm{Nd}$ :YAG pump laser which operated at a repetition of $10 \mathrm{~Hz}$. For the third piece of BBO crystal, 2.5 J second harmonic pulse, from an Nd:glass Subpicosecond Laser System (SPS), is utilized as the pump source [16]. The SPS laser is a two beam system, one beam is short with pulse width $\sim 1.0 \mathrm{ps}$ and pulse energy $100 \mathrm{~J}$. The other beam is a $1053 \mathrm{~nm}$ beam with the pulse width of about $2.0 \mathrm{~ns}$ and maximum pulse energy about $150 \mathrm{~J}$. The nanosecond beam is used as the pump source for our OPCPA system. The whole system works at one shot every thirty minutes. In the second stage, a piece of YCOB crystal with large aperture $\sim 50 \mathrm{~mm}$ is utilized as the gain medium and the SPS provides pulses at $526.5 \mathrm{~nm}$ with the desired special-temporal shape and energy exceeding $50 \mathrm{~J}$.

The signal is amplified in the XOZ principle of YCOB because the effective nonlinear coefficient $\left(d_{\mathrm{eff}}\right)$ in XOZ principle plane is much larger than that in XOY and YOZ principle planes $[17,18]$.

Noncollinear geometric configuration with type 1 phase matching $\mathrm{o}+\mathrm{o} \rightarrow \mathrm{e}$ is utilized in all OPA stages. Compared with the collinear geometric configuration, the noncollinear geometric configuration can achieve larger parametric bandwidth and get higher conversion efficiency by compensating

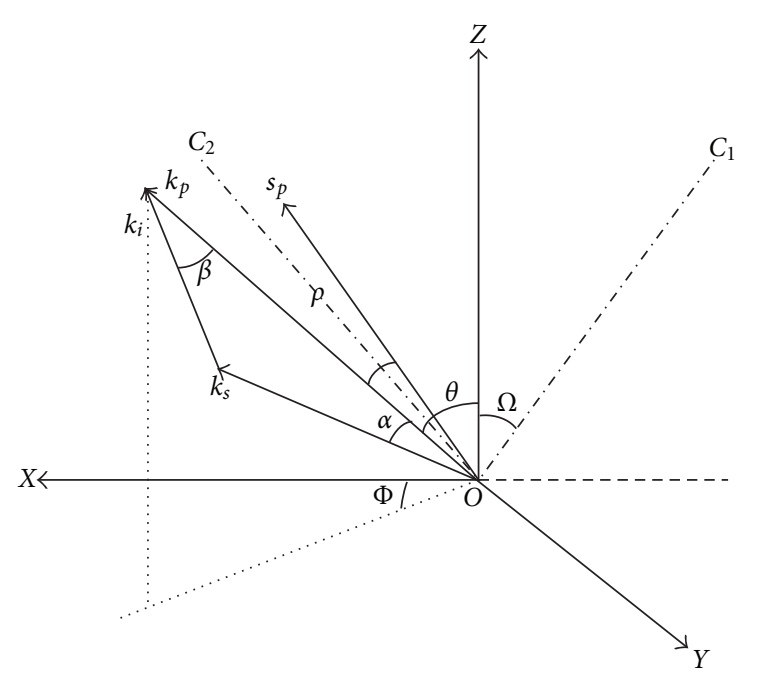

Figure 2: Noncollinear phase matching vectors in biaxial crystals.

walk-off effects of the pump pulse with noncollinear angle. Wave vectors and parameters of noncollinear phase matching in biaxial crystals are shown in Figure 2. $X, Y$, and $Z$ are principle axes. The refractive indexes along principle axes are ordered $n Z>n Y>n X . C_{1}$ and $C_{2}$ are optical axes. $\Omega$ is optic axial angle, $\Phi$ the azimuth of $k_{p}$ in XOZ principle plane, and $k_{s}, k_{i}$, and $k_{p}$ wave vectors of signal, idle, and pump, respectively. Symbol of $\alpha$ refers to the noncollinear angle, $\theta$ the phase matching angle, $\rho$ the walk-off angle, $s_{p}$ the Poynting vector of pump pulse, and $\beta$ the angle between $k_{i}$ and $k_{p}$. Momentum conservation and energy conservation during the OPA process can be expressed as (1) and (2). The wave vector mismatch is expressed as (3):

$$
\begin{gathered}
\vec{k}_{p}=\vec{k}_{i}+\vec{k}_{s}, \\
\omega_{p}=\omega_{i}+\omega_{s}, \\
\Delta k=k_{p}-k_{s} \cos \alpha-k_{i} \cos \beta .
\end{gathered}
$$

The optimized noncollinear configuration can realize the highest parametric bandwidth $\left(\Delta \lambda_{p}\right)$ for the ultrabroadband optical parametric amplifier. Generally speaking, 


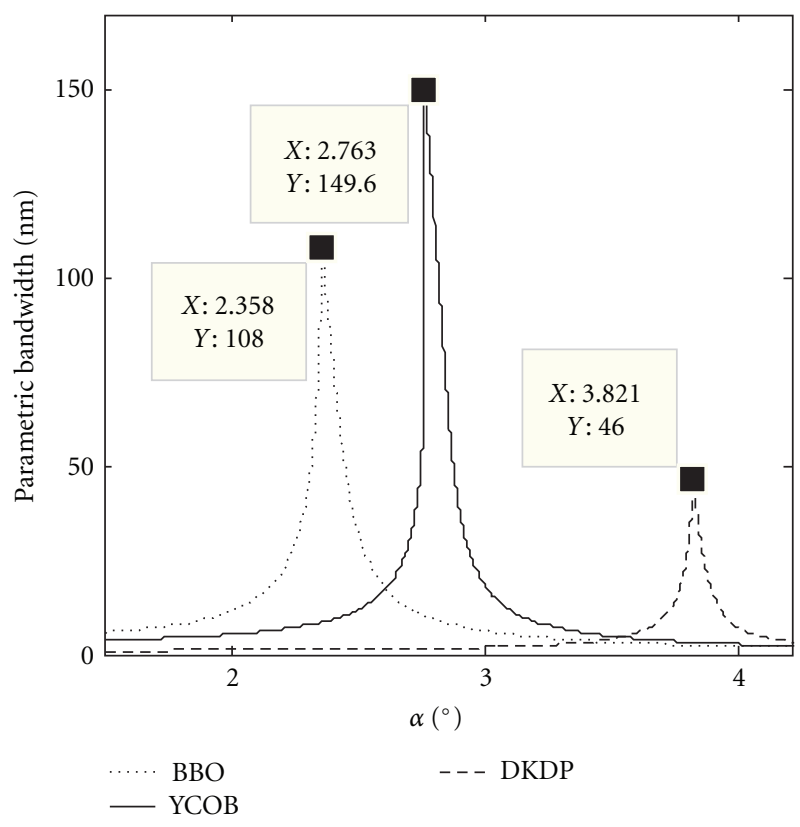

(a)

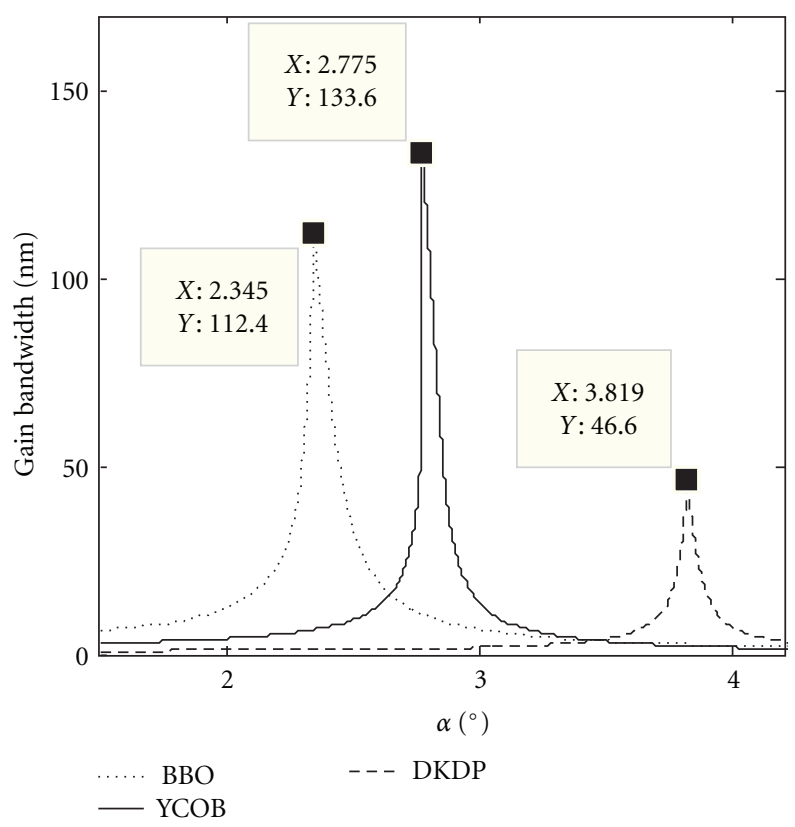

(b)

FIGURE 3: Parametric bandwidth (a) and gain bandwidth (b) versus noncollinear angles in BBO (dotted), in the XOZ principle plane of YCOB (solid), and in DKDP (dashed).

noncollinear angle in such configuration is set to realize group velocity matching (GVM) between signal pulse and pump pulse [19]. However, according to numerical analysis, there exists a small difference between the GVM noncollinear angle and the one for realizing the highest parametric bandwidth [20]. Parametric bandwidths as well as gain bandwidths $\left(\Delta \lambda_{g}\right)$ and acceptance angles $(\Delta \theta)$ are calculated exactly according to the definitions without using approximation of Taylor series. Identical analytic method was utilized in reference [18].

Parametric bandwidth is defined as the interval of the signal wavelength that restricts the phase mismatching smaller than $\pm \pi$ under the condition that perfect phase matching is achieved at the center wavelength of OPA. It can be expressed as $|\Delta k L| \leq \pi$, here $L$ is the crystal length. Figure 3(a) shows the curves of the parametric bandwidths versus noncollinear angles in $15 \mathrm{~mm} \mathrm{BBO}$ crystal, $15 \mathrm{~mm}$ YCOB crystal, and $35 \mathrm{~mm}$ DKDP. As shown in Figure 3(a), parametric bandwidths of $149.6 \mathrm{~nm}$ and $108 \mathrm{~nm}$ are achieved in YCOB and $\mathrm{BBO}$ when noncollinear angle are $2.763^{\circ}$ and $2.358^{\circ}$, respectively. The value is $46 \mathrm{~nm}$ in DKDP when noncollinear angle is $3.821^{\circ}$. Parametric bandwidth is consistent with the gain bandwidth to a large extent. Gain bandwidth is defined as the interval of the signal wavelength that restricts the gain bigger than half of that achieved at the center wavelength of signal pulse. As shown in Figure 3, the largest gain bandwidth of $133.6 \mathrm{~nm}$ is achieved at noncollinear angle of $2.775^{\circ}$ in YCOB, $112.4 \mathrm{~nm}$ at $2.345^{\circ}$ in $\mathrm{BBO}$, and $46.6 \mathrm{~nm}$ at $3.819^{\circ}$ in DKDP. As a conclusion, gain bandwidths of YCOB and $\mathrm{BBO}$ are broad enough for the amplification of chirped pulses, while DKDP is confided to the amplification of chirped pulses with original pulse duration exceeding $21 \mathrm{fs}$ at $808 \mathrm{~nm}$.
The walk-off effect results from the separation of Poynting vector and wave vector of light transmitting in nonlinear crystals (inhomogeneous medium) and leads to spatial displacement between the signal pulse and pump pulse and the degradation of conversion efficiency in OPCPA. In addition, walk-off effect plays an important role in the impact of wavefront phase distortion of pump on the beam quality of signal in OPA [21,22]. The acceptance angle is defined as the interval of $\theta$ that restricts the wave vector mismatch smaller than $\pm \pi / L$ around the perfect phase matching angle $\left(\theta_{\mathrm{pm}}\right)$ at $808 \mathrm{~nm}$. A large acceptance angle means a stable OPA. The walk-off angles, acceptance angles, and phase-matching angles versus noncollinear angles are shown in Figure 4. Corresponding to the optimal noncollinear angle, the walkoff angle in YCOB crystal is $1.134^{\circ}$, as well as acceptance angle $\sim 0.06^{\circ}$ and phase matching-angle $26.4^{\circ}$. The values of $\mathrm{YCOB}, \mathrm{BBO}$, and DKDP are listed in Table 1 and that the YCOB has bigger acceptance angle and smaller walk-off angle than $\mathrm{BBO}$ and DKDP.

As shown in Figure 5(a), for chirped signal pulse pumped by $532 \mathrm{~nm}$ pulse at intensity $\left(I_{p}\right)$ of $0.4 \mathrm{GW} / \mathrm{cm}^{2}$ in $15 \mathrm{~mm}$ BBO crystal, gain of 2732 can be realized. In the first two pieces of BBO crystals, unsaturated gain of $1000 \times 1000 \mathrm{can}$ be achieved by pumping with $532 \mathrm{~nm}$ pulse of $300 \mathrm{~mJ}$ at an intensity of $0.4 \mathrm{GW} / \mathrm{cm}^{2}$. In the third piece of $\mathrm{BBO}$ crystal, the injected signal pulse is amplified by 526.5 pump pulse with energy of $2.5 \mathrm{~J}$ at an intensity of $0.4 \mathrm{GW} / \mathrm{cm}^{2}$. The phase matching parameters listed in Table 1 are calculated with the same method. There are not big differences with those pumped by pulse at $532 \mathrm{~nm}$. The BBO crystal utilized in this stage is $13 \mathrm{~mm}$ (aperture) $\times 15 \mathrm{~mm}$ (length) to realize saturated gain of $\sim 2000$ so that energy of signal pulse is amplified up to $\sim 0.4 \mathrm{~J}$. The majority of energy is provided 


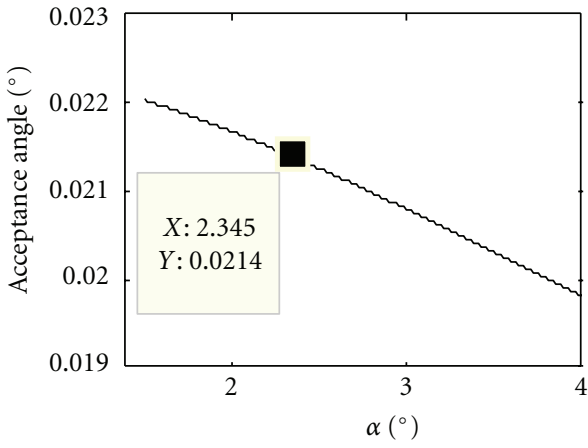

(a)

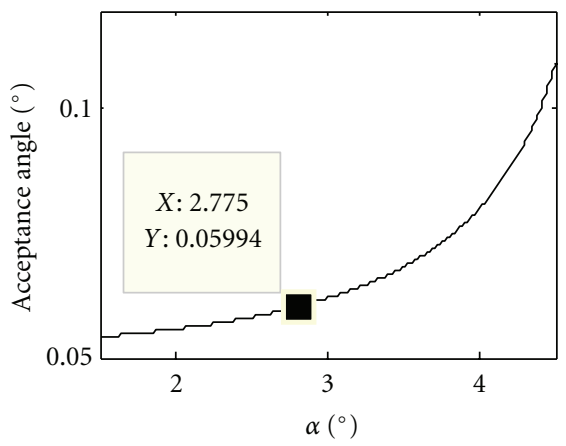

(c)

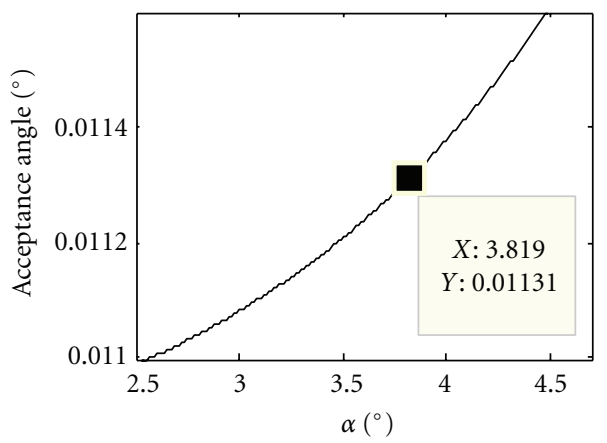

(e)

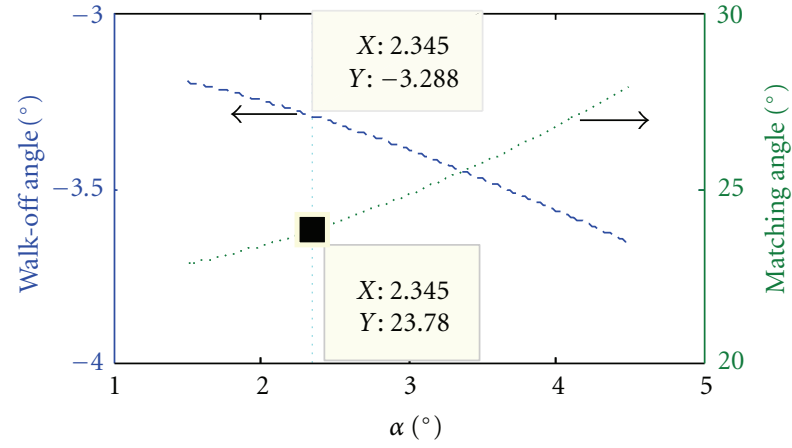

(b)

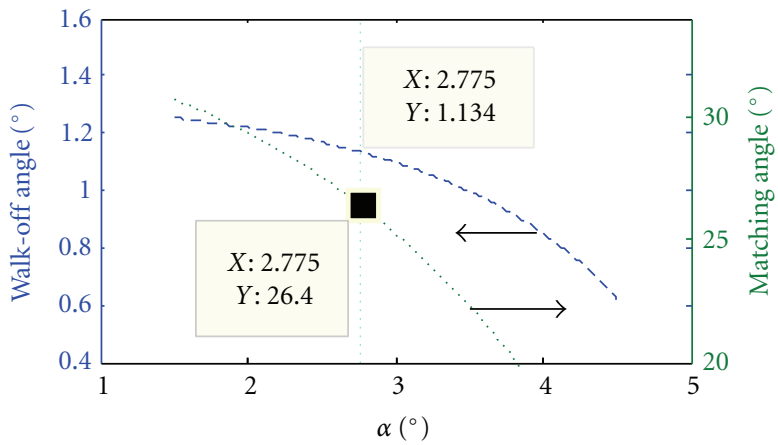

(d)

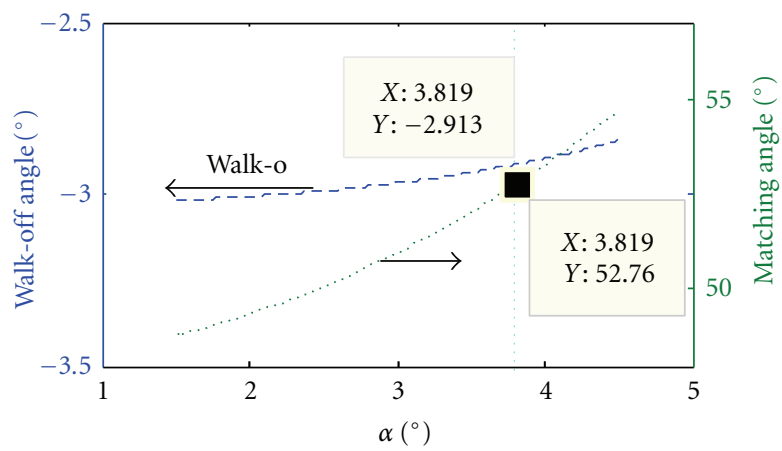

(f)

FIGURE 4: Acceptance angle (solid), walk-off angle (dashed), and phase-matching angle (dotted) versus noncollinear angle in BBO (a and b), the XOZ principle plane of YCOB (c and d), and DKDP (e and f).

TABLE 1: Noncollinear phase matching parameters of BBO, YCOB, and DKDP.

\begin{tabular}{lccccccccc}
\hline & $\alpha\left(^{\circ}\right)$ & $\theta_{\mathrm{pm}}\left({ }^{\circ}\right)$ & $\Delta \lambda_{p}(\mathrm{~nm})$ & $d_{\text {eff }}(\mathrm{pm} / \mathrm{V})$ & $\rho\left(^{\circ}\right)$ & $\Delta \theta\left(^{\circ}\right)$ & $\Delta \lambda_{g}(\mathrm{~nm})$ & $I_{p}\left(\mathrm{GW} / \mathrm{cm}^{2}\right)$ & $L(\mathrm{~m})$ \\
\hline BBO-1/2 & 2.345 & 23.78 & 108 & 2.096 & -3.288 & 0.0214 & 112.4 & 0.4 & 0.015 \\
BBO-3 & 2.4 & 23.86 & 132 & 2.095 & -3.295 & 0.0214 & 127 & 0.4 & 0.015 \\
\hline YCOB & 2.775 & $(26.4,180)$ & 149.6 & 0.935 & 1.134 & 0.0599 & 133.6 & 0.5 & 0.015 \\
\hline DKDP & 3.819 & 52.76 & 46 & 0.295 & -2.913 & 0.0113 & 46.6 & 2 & 0.035 \\
\hline
\end{tabular}

by the second stage. Figure 5(b) shows the gain curve in the XOZ principle plane of YCOB with $526.5 \mathrm{~nm}$ pump pulse. For YCOB crystal of $15 \mathrm{~mm}$ in length, saturated gain of $\sim 20$ can be realized with pump pulse at intensity of $0.5 \mathrm{GW} / \mathrm{cm}^{2}$ and energy of $50 \mathrm{~J}$. In the power amplification stage, signal pulse of $\sim 10 \mathrm{~J}$ can be achieved with broad spectral bandwidth. Moreover, saturated amplification in both stages would greatly improve the stability of the system [23]. Normalized gain curve in $35 \mathrm{~mm}$ DKDP is also shown in Figure 5(a) when pumped by $526.5 \mathrm{~nm}$ pulse at intensity of $2 \mathrm{GW} / \mathrm{cm}^{2}$. DKDP is a good choice for amplifying pulses centered at $808 \mathrm{~nm}$ with duration exceeding $20 \mathrm{fs}$.

As the temporal profile is an important factor in the ultrashort laser mater interaction experiment, a spectrum 


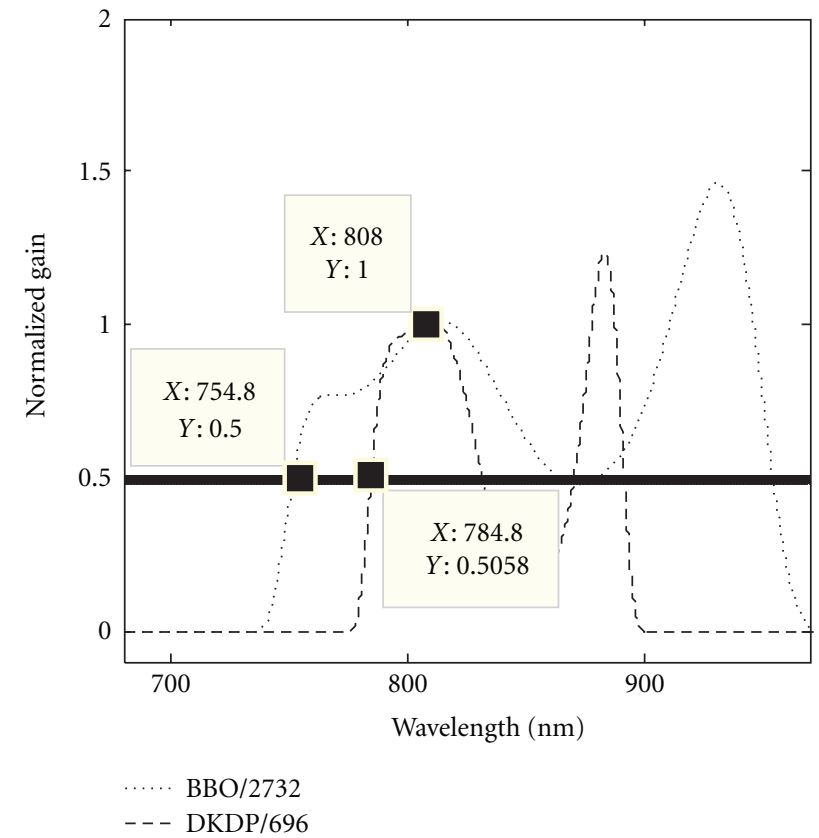

(a)

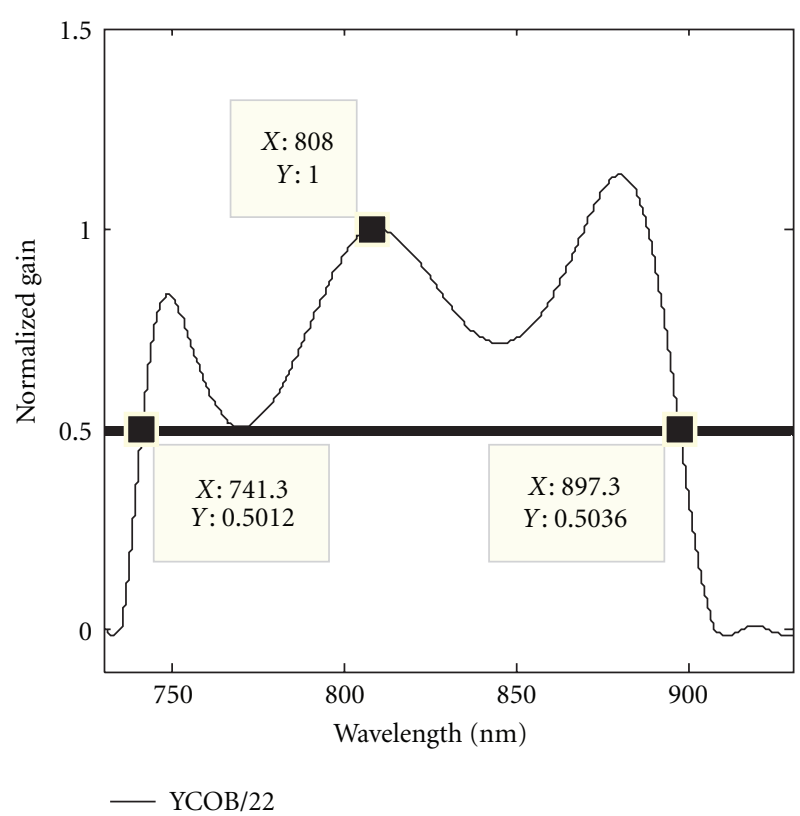

(b)

FIGURE 5: Normalized gain curves in BBO (a) (dotted), DKDP (a) (dashed), and XOZ principle plane of YCOB crystal (b) (solid).

shaper or a Dazzler with the resolution of $0.6 \mathrm{~nm}$ is used within the first OPCPA stage to control the pulse envelop. A 39-element deformable mirror with diameter of $50 \mathrm{~mm}$ is used before the pulse compression to ensure a good beam quality and the shortest pulse width.

The compressor is consisted of a pair of gratings and a piece of reflector. They are arranged in tandem in a fourpass configuration with Littrow incident angle of $36.3^{\circ}$ to match with the stretcher so that the chirped pulse can be compressed to Fourier transform limitation. The transmission efficiency of the compressor is $~ 75 \%$ and the energy of output ultrashort pulse would be $\sim 8 \mathrm{~J}$.

In conclusion, a new type of OPCPA system to realize the amplification of pulse centered at $808 \mathrm{~nm}$ is analyzed, and noncollinear phase matching parameters of YCOB is compared with those of BBO and DKDP in Table 1. Numerical analysis shows that YCOB rather than DKDP can be utilized to amplify ultrabroadband chirped pulse so that the signal pulse can be compressed to about $10 \mathrm{fs}$ after the compressor. With YCOB as the gain medium of OPCPA, a total gain of $\sim 4 \times 10^{10}$ is achieved and the energy of the amplified signal pulse is up to several joules.

\section{References}

[1] Y. Nomura, R. Hörlein, P. Tzallas et al., "Attosecond phase locking of harmonics emitted from laser-produced plasmas," Nature Physics, vol. 5, no. 2, pp. 124-128, 2009.

[2] F. Quere, "Attosecond plasma optics," Nature Physics, vol. 5, pp. 93-94, 2009.

[3] D. Charalambidis, P. Tzallasl, E. P. Benis et al., "Exploring intense attosecond pulses," New Journal of Physics, vol. 10, Article ID 025018, 2008.
[4] G. D. Tsakiris, K. Eidmann, J. Meyer-ter-Vehn, and F. Krausz, "Route to intense single attosecond pulses," New Journal of Physics, vol. 8, Article ID 025018, 2006.

[5] E. Hugonnot, G. Deschaseaux, O. Hartmann, N. Beck, and H. Coïc, "Optical parametric chirped pulse amplification for the PETAL front-end: design and first results," in Proceedings of the Advanced Solid-State Photonics (ASSP '07), OSA Technical Digest Series (CD), p. MB4, Optical Society of America, Vancouver, Canada, 2007.

[6] M. Sun, J. Kang, A. Guo et al., "Effect of single-mode fiber on the contrast of chirped-pulse," Acta Optica Sinica, vol. 30, no. 5, pp. 1234-1237, 2010.

[7] E. Gaul, M. Martinez, J. Blakeney et al., "Activation of a 1.1 Petawatt hybrid, OPCPA-Nd: glass laser," in Proceedings of the Conference on Lasers and Electro-Optics and the Conference on Quantum Electronics and Laser Science Conference (CLEO/QELS '09), June 2009.

[8] Y. Kitagawa, H. Fujita, R. Kodama et al., "Prepulse-free Petawatt laser for a fast ignitor," IEEE Journal of Quantum Electronics, vol. 40, no. 3, pp. 281-293, 2004.

[9] C. N. Danson, P. A. Brummitt, R. J. Clarke et al., "Vulcan Petawatt-an ultra-high-intensity interaction facility," Nuclear Fusion, vol. 44, no. 12, pp. S239-S246, 2004.

[10] R. Norrestam, M. Nygren, and J. O. Bovin, "Structural investigations of new calcium-rare earth $(\mathrm{R})$ oxyborates with the composition $\mathrm{Ca}_{4} \mathrm{RO}\left(\mathrm{BO}_{3}\right)_{3}$," Chemistry of Materials, vol. 4, no. 3, pp. 737-743, 1992.

[11] Z. Wang, K. Fu, X. Xu et al., "The optimum configuration for the third-harmonic generation of $1.064 \mu \mathrm{m}$ in a YCOB crystal," Applied Physics B, vol. 72, no. 7, pp. 839-842, 2001.

[12] M. Iwai, T. Kobayashi, H. Furuya, Y. Mori, and T. Sasaki, "Crystal growth and optical characterization of rare-earth ( $\mathrm{Re}$ ) calcium oxyborate $\mathrm{ReCa}_{4} \mathrm{O}\left(\mathrm{BO}_{3}\right)_{3}(\mathrm{Re}=\mathrm{Y}$ or $\mathrm{Gd})$ as new nonlinear optical material," Japanese Journal of Applied Physics: Part 2, vol. 36, no. 3, pp. L276-L279, 1997. 
[13] C. A. Ebbers, A. J. Bayramian, R. W. Campbell et al., "High average power frequency conversion with large aperture YCOB," in Proceedings of the Advanced Solid-State Photonics (ASSP '08), OSA Technical Digest Series (CD), p. WD3, Optical Society of America, Nara, Japan, January 2008.

[14] Z. M. Liao, I. Jovanovic, C. A. Ebbers, Y. Fei, and B. Chai, "Energy and average power scalable optical parametric chirped-pulse amplification in yttrium calcium oxyborate," Optics Letters, vol. 31, no. 9, pp. 1277-1279, 2006.

[15] O. V. Chekhlov, J. L. Collier, I. N. Ross et al., "35 J broadband femtosecond optical parametric chirped pulse amplification system," Optics Letters, vol. 31, no. 24, pp. 3665-3667, 2006.

[16] X. L. Xie, J. Q. Zhu, F. Q. Liu et al., "20 TW sub-picosecond laser system (SPS) applied for the neutron experiment," Chinese Journal of Lasers, vol. 30, no. 10, pp. 865-872, 2003.

[17] M. V. Pack, D. J. Armstrong, A. V. Smith, G. Aka, B. Ferrand, and D. Pelenc, "Measurement of the $X^{(2)}$ tensor of $\mathrm{GdCa}_{4} \mathrm{O}\left(\mathrm{BO}_{3}\right)_{3}$ and $\mathrm{YCa} \mathrm{O}_{4} \mathrm{O}\left(\mathrm{BO}_{3}\right)_{3}$ crystals," Journal of the Optical Society of America B, vol. 22, no. 2, pp. 417-425, 2005.

[18] M. Sun, Q. Bi, F. Zhang, J. Kang, X. Xie, and Z. Lin, "Study on noncollinear phase matching in YCOB crystal," Acta Optica Sinica, vol. 31, no. 1, Article ID 0119001, 2011.

[19] T. Wilhelm, J. Piel, and E. Riedle, "Sub-20-fs pulses tunable across the visible from a blue-pumped single-pass noncollinear parametric converter," Optics Letters, vol. 22, no. 19, pp. 1494-1496, 1997.

[20] J. Wang, J. Q. Yao, X. F. Li, Y. Z. Yu, and X. C. Shi, "Amendatory calculation of the acceptance parameters in three-wave interactions," Acta Optica Sinica, vol. 21, no. 2, pp. 139-141, 2001.

[21] F. Zhang, Y. Wang, M. Sun, Q. Bi, X. Xie, and Z. Lin, "Numerical simulations of the impact of wavefront phase distortions of pump on the beam quality of OPA," Chinese Optics Letters, vol. 8, no. 2, pp. 217-220, 2010.

[22] X. Wei, L. Qian, P. Yuan, H. Zhu, and D. Fan, "Optical parametric amplification pumped by a phase-aberrated beam," Optics Express, vol. 16, no. 12, pp. 8904-8915, 2008.

[23] J. Kang, S. H. Chen, J. Q. Zhu, and H. Wei, "Design of a highly stable, high-conversion-efficiency pumping source for optical parametric amplifier by extending efficient crystal length," Optics and Laser Technology, vol. 39, no. 5, pp. 1084-1088, 2007. 

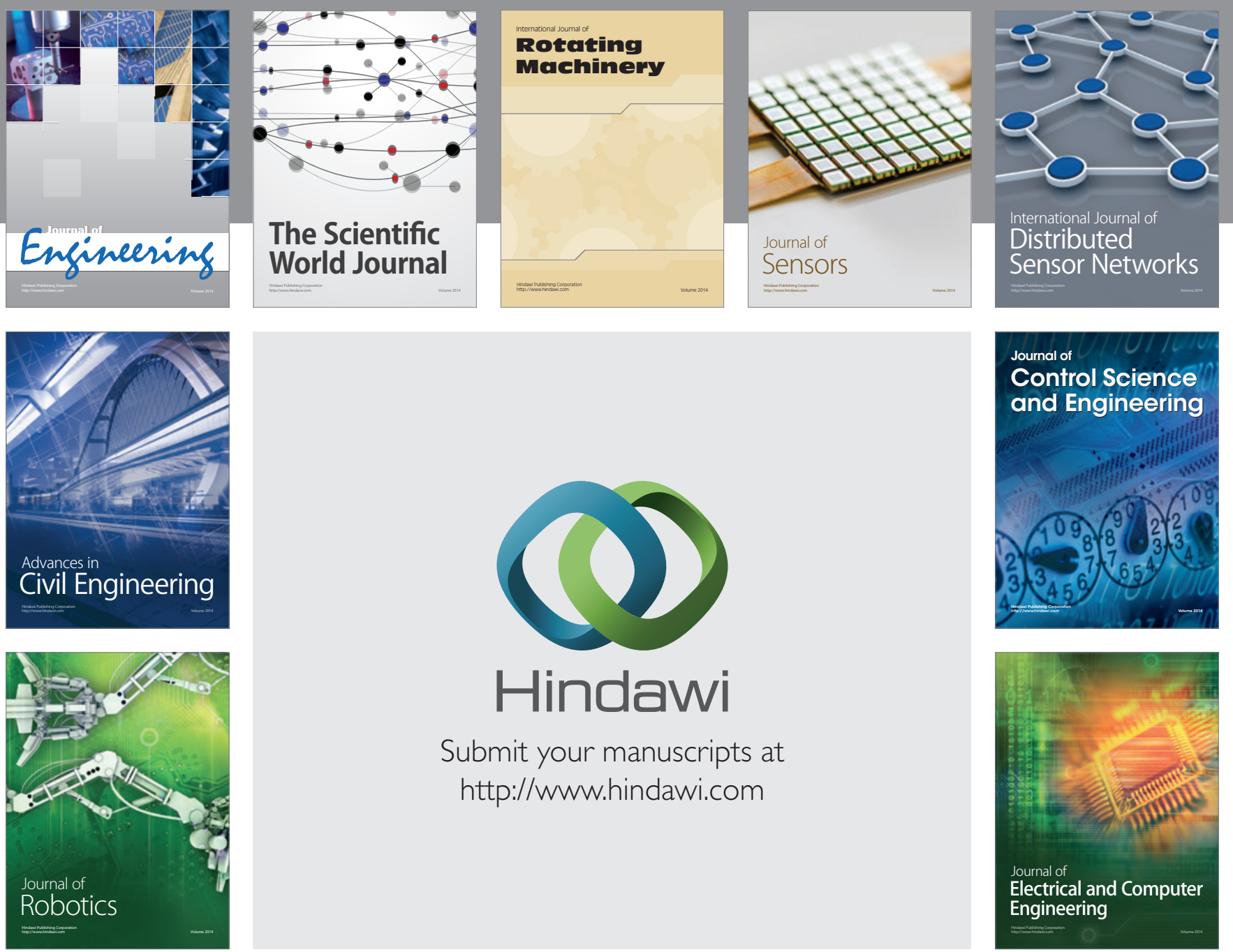

Submit your manuscripts at

http://www.hindawi.com
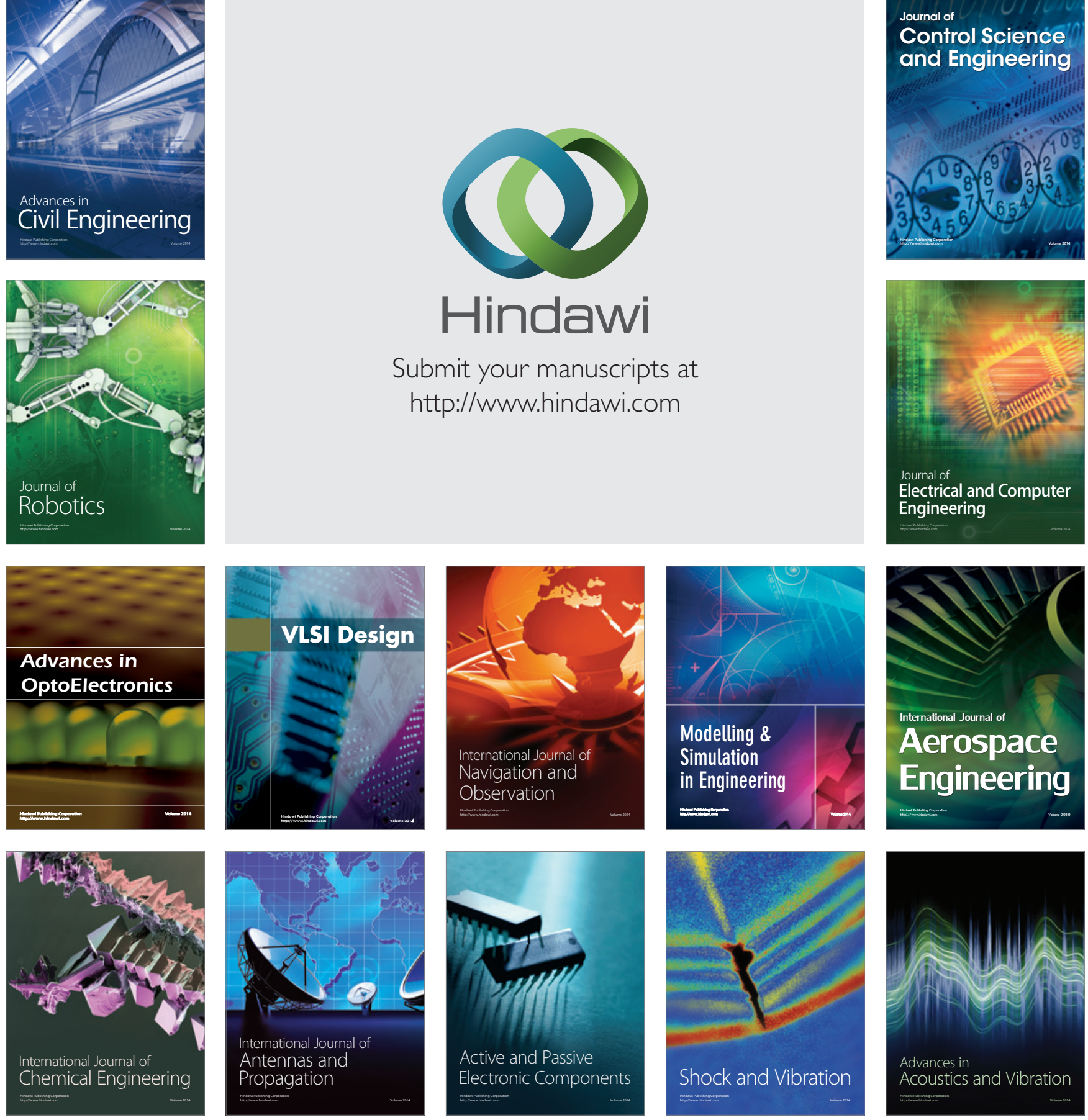